\title{
Assessment of geometrical characteristics of dental endodontic micro-instruments utilizing X-ray micro computed tomography
}

\author{
Youssef S. AI JABBARI' ${ }^{1}$, Peter TSAKIRIDIS², George ELIADES ${ }^{3}$, Solaiman M. AL-HADLAQ ${ }^{4}$, Spiros ZINELIS 5,6 \\ 1- BDS, MS, PhD, Director, Dental Biomaterials Research and Development Chair, College of Dentistry, King Saud University, Riyadh, Saudi Arabia. \\ 2- Research Assistance, Department of Biomaterials, School of Dentistry, University of Athens, Athens, Greece. \\ 3- DDS, PhD, FADM, Professor, Department of Biomaterials, School of Dentistry, University of Athens, Athens, Greece. \\ 4- BDS, MS, PhD, Professor, Dental Biomaterials Research and Development Chair, College of Dentistry, King Saud University, Riyadh, Saudi Arabia. \\ 5- PhD, FADM, Assistante Professor, Department of Biomaterials, School of Dentistry, University of Athens, Athens, Greece. \\ 6- Consultant, Dental Biomaterials Research and Development Chair, College of Dentistry, King Saud University, Riyadh, Saudi Arabia.
}

Corresponding address: Dr. Youssef S. Al Jabbari, BDS. MS. PhD. - Director, College of Dentistry Research Center - Director, Dental Biomaterials Research and Development Chair - Department of Prosthetic Dental Sciences, King Saud University College of Dentistry - P.O.Box 60169 - Riyadh 11545 - Saudi Arabia - Phone: +966 14698312 - Fax: +966 14698313 - e-mail: yaljabbari@ksu.edu.sa

Received: April 01, 2012 - Modification: August 23, 2012 - Accepted: October 23, 2012

\section{ABSTRACT}

$\mathrm{O}$ bjective: The aim of this study was to quantify the surface area, volume and specific surface area of endodontic files employing quantitative X-ray micro computed tomography $(\mathrm{mXCT})$. Material and Methods: Three sets (six files each) of the Flex-Master $\mathrm{Ni}$-Ti system (No 20, 25 and 30, taper .04) were utilized in this study. The files were scanned by $\mathrm{mXCT}$. The surface area and volume of all files were determined from the cutting tip up to $16 \mathrm{~mm}$. The data from the surface area, volume and specific area were statistically evaluated using the one-way ANOVA and SNK multiple comparison tests at $\alpha=0.05$, employing the file size as a discriminating variable. The correlation between the surface area and volume with nominal ISO sizes were tested employing linear regression analysis. Results: The surface area and volume of No 30 files showed the highest value followed by No 25 and No 20 and the differences were statistically significant. The No 20 files showed a significantly higher specific surface area compared to No 25 and $N^{\circ} 30$. The increase in surface and volume towards higher file sizes follows a linear relationship with the nominal ISO sizes ( $r^{2}=0.930$ for surface area and $r^{2}=0.974$ for volume respectively). Results indicated that the surface area and volume demonstrated an almost linear increase while the specific surface area exhibited an abrupt decrease towards higher sizes. Conclusions: This study demonstrates that $\mathrm{mXCT}$ can be effectively applied to discriminate very small differences in the geometrical features of endodontic micro-instruments, while providing quantitative information for their geometrical properties.

Key words: X-ray microtomography. Nickel. Titanium. Dental instruments.

\section{INTRODUCTION}

X-ray micro computed tomography ( $\mathrm{MXCT}$ ) has many applications in various fields including medicine, geology, materials science, dentistry, art and archaeology, among others $\mathrm{s}^{4,5,10,13}$. The methodology combines a non-destructive and noninvasive analysis with the ability to quantify the geometrical characteristics of irregular specimens such as bones, archaeological remains, teeth, etc. The technique is constantly being improved through hardware and software developments ${ }^{10}$ and the subsequent increase in resolution provides researchers with new information and approaches for the investigation of the material properties.

In the dental field, $\mathrm{mXCT}$ has been extensively used to quantify the geometrical changes in the root canal anatomy during endodontic therapy, comparing pre and post instrumentation analyses ${ }^{11,15,18,19,21}$. Root canals receive a chemo-mechanical treatment with successively larger endodontic files (Figure 1 ) in order to prepare the anatomy and surface of 
the root canal to support the dental posts. This is a very demanding task as endodontic files must cut the dentin and remove the dental debris, preserving the curvature of the root canals without fracture. Root fracture is the biggest complication during instrumentation, jeopardizing the outcome of endodontic therapy ${ }^{20,24}$. Despite the evolution of endodontic files over the last four decades and the large number of available endodontic systems, no system is free of problems and drawbacks ${ }^{16}$.

Endodontic files are made from different grades of austenitic stainless steel ${ }^{3}$ or $\mathrm{Ni}^{-\mathrm{Ti}^{29}}$ alloys characterized by a variety of geometrical features such as taper, cross-section, helix and rake angle, and the distance between the successive cutting blades ${ }^{27}$. These features are critical factors in the mechanical resistance of endodontic files to load forces (i.e., bending and torsion), their cutting capacity, and the clearance of dental chips $7,22,26,28$. The introduction of the $\mathrm{Ni}$ $\mathrm{Ti}$ alloy in file manufacturing was followed by the

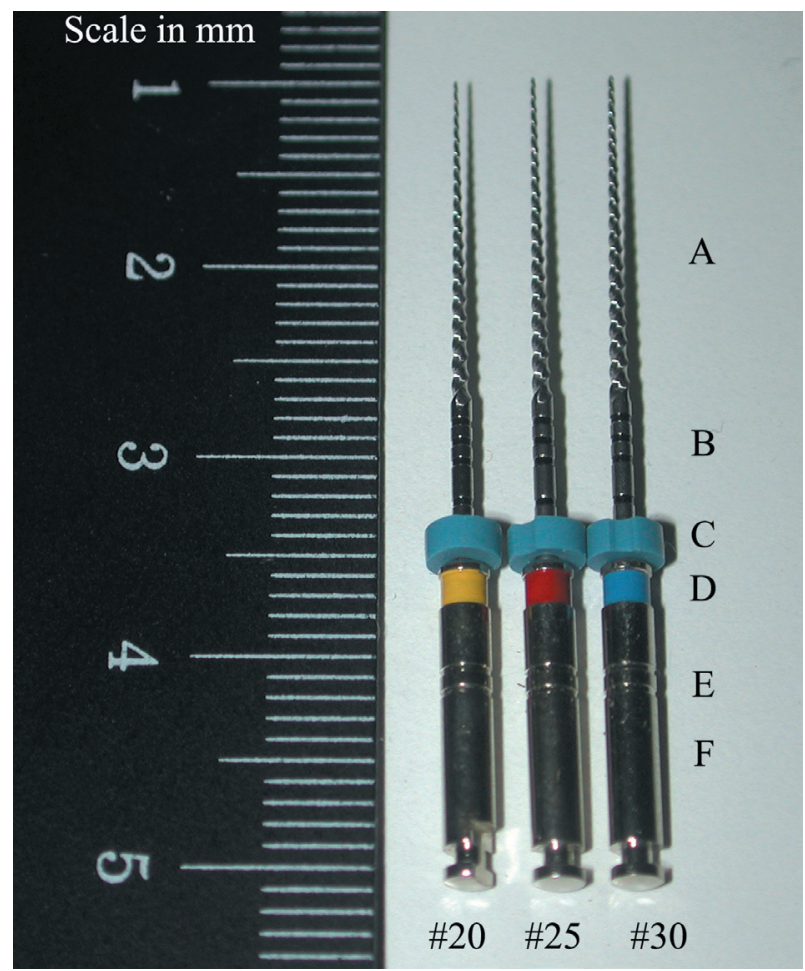

Figure 1- Macroscopic view of the endodontic files (FlexMaster) used in this study. They appear similar to the naked eye. A: The cutting portion. B: Depth marking facilitates determination of the exact position of the file on x-ray. C: Silicon rubber for working length marking. Color coding indicated according to ISO requirements (Yellow: № 20, Red: № 25, Blue: $N^{\circ} 30$.). E: The number of engraved rings on the shaft determines the taper of the instrument. One ring represents taper .02, 2 rings for .04 and 3 rings for taper .06. F: Specially designed shaft to be fitted in the handle of the low torque motor. The scale on the left is in $\mathrm{mm}$ development of a variety of new cross-sectional designs with an increased taper compared to the 0.02 employed in $\mathrm{K}$ - and $\mathrm{H}$-type files made of stainless steel as dictated by the International Standard Organization (ISO) specification $3630^{9,30}$. Nevertheless, the determination of other important geometrical features such as the surface area and volume of the endodontic files remain unknown, limiting the assessment of other parameters derived from these basic features. Precise estimation of the basic geometrical features might provide an additional approach to explain the differences in the clinical performance among various file designs and establish a valuable tool for development of new and more efficient geometrical designs.

Apart from the K-files, where the surface area and volume can be calculated based on the untwisted tapered blank values employing mathematical formulas, the calculation of these features was impossible before the introduction of $\mathrm{mXCT}$. This technique has been extensively used to determine the geometrical features, such as shape, surface area and the volume of the root canals before and after treatment with endodontic instruments $2,11,14,18,21$ but has never been utilized to determine the geometrical features of endodontic files.

The aim of this study was to determine, for the first time, the surface area, volume and specific surface area of successive file sizes of a commercially available $\mathrm{Ni}-\mathrm{Ti}$ system. Additionally, we tested the hypothesis that $\mathrm{mXCT}$ is capable of discriminating the quantitative differences in the aforementioned geometrical properties between the successive sizes of endodontic files. These differences exist by definition due to the progressively increased size of the successive files used in root canal therapy. The hypothesis tested was that there are significant differences in the surface area, volume and specific surface area among the different sizes of endodontic files.

\section{MATERIAL AND METHODS}

Three sets of Flex-Master Ni-Ti files (VDW; Munich, Germany), 6 files each (ISO sizes No 20/ Lot 0411310306, No 25/Lot 0410310302 and No 30/Lot 0406310290) all of 0.04 taper, were utilized in this study. To avoid any inclination from the vertical axis, the files were placed on the stage, employing a custom made attachment and then scanned by a mXCT scanner (SkyScan Model 1072; Aartselaar, Belgium) operated under the following conditions: Wo Ka source $(100 \mathrm{kV}, 98 \mu \mathrm{A}), 2.36$ $\mu \mathrm{m}$ pixel size, $180^{\circ}$ rotation, 0.9 rotation step, 1.9 sec exposure time averaging by two frames, and $1 \mathrm{~mm}$ Al filter. The surface area and volume of all files were determined from the tip up to the 16 
mm level, taking 6,782 horizontal slices. The files were successively scanned using the automated routine of oversize mode, for $16 \mathrm{~h}$ each. A fixed threshold was applied to discriminate the crosssection from the air, providing high contrast images of the horizontal cross-sections. The reconstruction was made employing a cone beam reconstruction (Feldkam) algorithm. The surface area (SA) and volume (V) of each file were determined by the embedded software (CTAN, SkyScan, Aartselaar, Belgium) employing the following formulas:

$$
\begin{aligned}
& \mathrm{SA}=\sum_{i=1}^{n} p_{i} s \\
& \mathrm{~V}=\sum_{i=1}^{n} a_{i} s
\end{aligned}
$$

with $n=$ number of slices, $s=$ slice thickness, $\mathrm{p}_{\mathrm{i}}=$ measured periphery in slice $\mathrm{i}$ and $\mathrm{a}_{\mathrm{i}}=$ measure area in slice $i$

The specific surface area (the surface to volume ratio) was also calculated. The percentage differences between the successive file sizes for the aforementioned properties were calculated based on the formula:

$$
\% \text { Dif }=100 *\left(S_{n}-S_{n-1}\right) / S_{n-1}
$$

Horizontal slices of all scanned files were also inspected for the presence of internal defects such as cracks or pores.

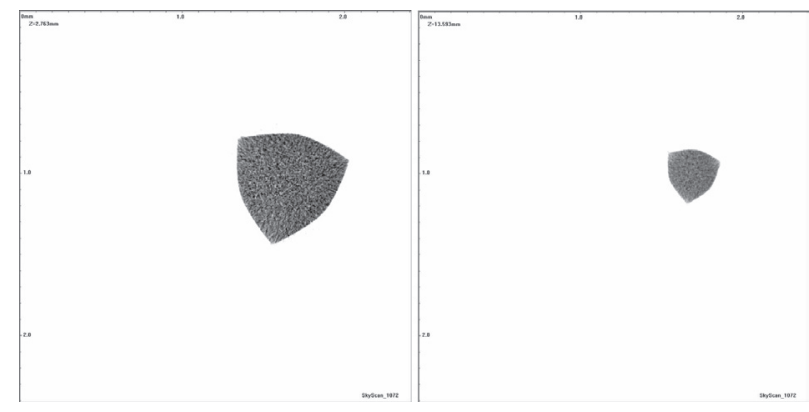

Figure 2- Left and right images show two horizontal cross sections of the same file. The distance between crosssections is $11.830 \mathrm{~mm}$. The right image has a smaller cross- sectional surface, as it is closer to the cutting tip

\section{Statistical analysis}

All data were statistically analyzed using oneway ANOVA and SNK multiple comparison tests at a 95\% significance level, employing the file size as the discriminating variable. Linearity of the surface area and volume data versus ISO size was analyzed employing linear regression analysis.

\section{RESULTS}

Figure 2 demonstrates two representative horizontal sections (perpendicular to the longitudinal file axis) from the $\mathrm{mXCT}$. The left image has been taken from a region near the handle and the right from a region near the cutting tip of a $\mathrm{Ni}-\mathrm{Ti}$ file, demonstrating the difference in the cross-sectional surface area. The cross-section was found to be free of internal defects such as pores or cracks.

The quantitative results for the surface area, volume and specific surface area of successive file sizes are presented in Table 1 . The surface area of the $\mathrm{N}^{\circ} 30$ files showed the highest value followed by No 25 and No 20. Statistical analysis demonstrated significant differences among all the files sizes tested. The increase in total surface area was $8.9 \%$ between No 20/No 25 and $16.6 \%$ between No $25 /$ No 30 files, demonstrating close to a $100 \%$ increase for the latter. Similar results were found for the volume of the $\mathrm{Ni}$-Ti files tested. However, the increase in volume declined towards the larger file sizes. The percentage increase was estimated as $21.1 \%$ between No 20/No 25 and $17.2 \%$ between No 25/No 30). The file No 20 showed a significantly higher specific surface area followed by No 25 and No 30. The latter showed no statistically significant differences between them. The specific surface area showed a $10.1 \%$ decrease between No 20/No 25, but only $0.4 \%$ between No 25/NO 30 . Regression analysis revealed a strong linearity between the surface area (Figure 3a) and volume (Figure 3b) versus file sizes. The raw data, the fitted line, the $95 \%$ confidence intervals, the yielded analytical formula, and the regression coefficient $\left(r^{2}\right)$ are all presented in Figures $3 \mathrm{a}$ and $3 \mathrm{~b}$ for the surface area and volume, respectively.

Table 1- Mean values and standard deviations for the surface area (SA), volume (V), and specific surface area (SSA) of FlexMaster Ni-Ti files. The percentage differences (Dif) between successive file sizes are also presented

\begin{tabular}{ccccccc}
\hline ISO No & SA & Dif & V & Dif & SSA & Dif \\
& $\left(\mathrm{mm}^{2}\right)$ & $(\%)$ & $\left(\mathrm{mm}^{3}\right)$ & $(\%)$ & $\left(\mathrm{mm}^{-1}\right)$ & $(\%)$ \\
\hline 20 & $27.99 \pm 0.98^{\mathrm{a}}$ & & $2.58 \pm 0.10^{\mathrm{a}}$ & & $10.86 \pm 0.80^{\mathrm{a}}$ & \\
25 & $30.50 \pm 0.73^{\mathrm{b}}$ & 8,9 & $3.13 \pm 0.07^{\mathrm{b}}$ & 21,1 & $9.76 \pm 0.13^{\mathrm{b}}$ & $-10,1$ \\
30 & $35.58 \pm 1.15^{\mathrm{c}}$ & 16,6 & $3.66 \pm 0.06^{\mathrm{c}}$ & 17,3 & $9.71 \pm 0.47^{\mathrm{b}}$ & $-0,5$ \\
\hline
\end{tabular}

The same superscripts indicate mean values without statistical significant differences $(p>0.05)$ 

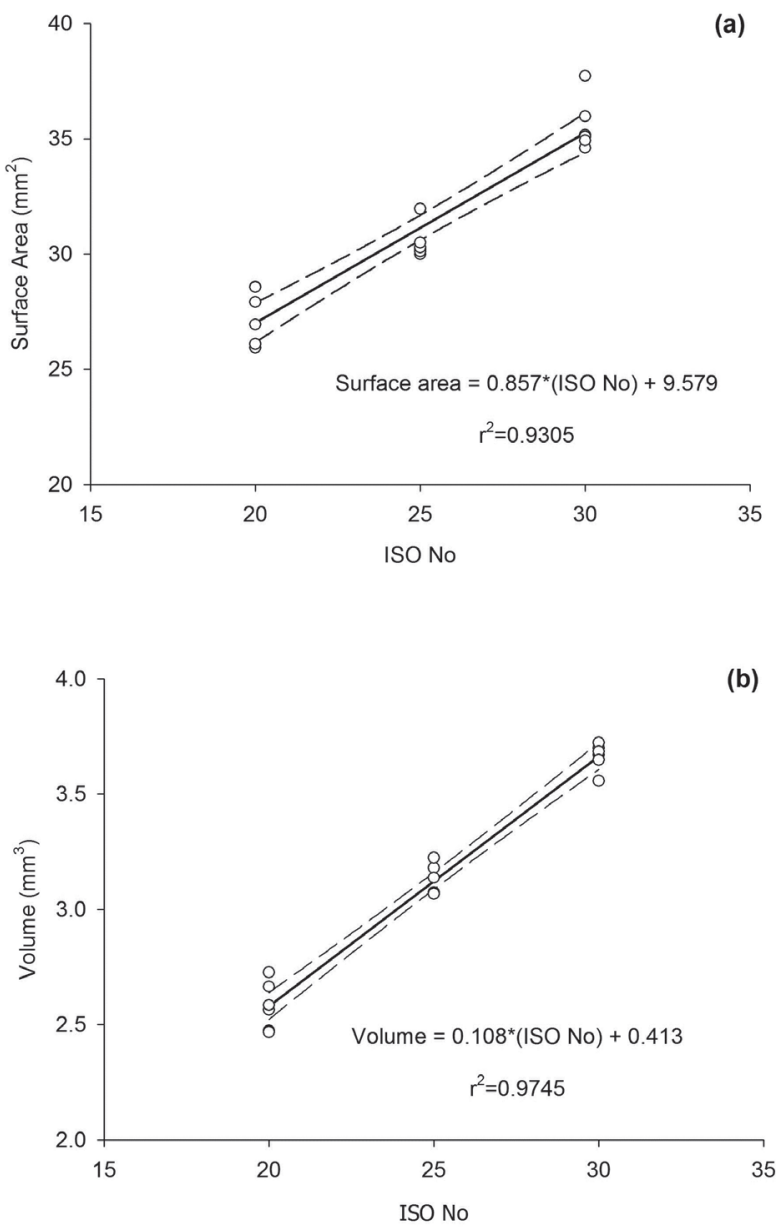

Figure 3- Raw data, fitted line with the 95\% confidence intervals, mathematical equations and liner regression coefficients $\left(r^{2}\right)$, for the surface area (a) and volume (b) of the FlexMaster Ni-Ti system

\section{DISCUSSION}

The results of the current study showed significant differences for the surface area and volume between the files tested, thus the original hypothesis is accepted. It is noteworthy that this analysis provided significant differences for the endodontic files with different ISO sizes, demonstrating the ability of this analysis to discriminate the quantitative differences of the geometrical characteristics among the different endodontic file sizes. This discrimination has also been facilitated due to the increased resolution of $2.36 \mu \mathrm{m}$ obtained in this study. The transition to scanner technology has a beneficial effect on the resolution of $\mathrm{mXCT}$ from $1000 \mu \mathrm{m}$ for conventional tomography, which was insufficient for endodontic applications ${ }^{6,14}$, to roughly $30 \mu \mathrm{m}^{8,17}$, providing the ability for more extensive research, especially in the root canal anatomy after chemo-mechanical treatments ${ }^{15,19}$. Currently available mXCT scanners can provide a maximum resolution up to $0.2 \mu \mathrm{m}$. However, beyond the technological advancement, the resolution of a specific analysis with a given $\mathrm{mXCT}$ scanner is limited by the size of the object, as it is magnification dependent. This is a disadvantage in addition to the restriction that all horizontal projections produced during rotation must be fitted to the maximum horizontal width of the CCD camera. Therefore, larger specimens must be scanned using lower magnifications, compromising resolution. The $\mathrm{mXCT}$ device used in this study has a maximum isotropic resolution of $1.8 \mu \mathrm{m}$ but the maximum achievable isotropic resolution for endodontic file scanning remained at $2.36 \mu \mathrm{m}$, due to dimensional constraints. The scanning of an alloy with a homogeneous elemental atomic ratio ${ }^{29}$ and a uniform X-ray absorption through its bulk facilitates image contrast and thus the selection of the proper threshold level to discriminate the edges of the cross-sections. All tested files were found free of internal defects such as pores or cracks, a finding which is in agreement with previous cross-sectional and $\mathrm{mXCT}$ analyses ${ }^{23,29}$.

Despite the increased isotropic resolution available through technological advancements, the accuracy of the quantitative analysis remains unknown, at least for endodontic applications dealing with the quantification of the geometrical features of the root canals and endodontic files. It is logical to assume that this accuracy is dependent on the isotropic resolution and might increase towards smaller isotropic resolutions. However, such an approach requires the development of specific standards made of the same material as the unknown sample, in order to preserve the same contrast. This could be a milestone for the application of $\mathrm{mXCT}$ in the quantification of the geometrical features and could be an interesting subject for future research.

The surface area and volume of the endodontic files, as presented for the first time by the present study, was found to increase towards higher file sizes with significant differences between the successive file sizes (Table 1 ). Linear regression analysis showed a linear correlation of both the surface area and volume with the nominal file sizes. The surface area and volume are not physically dependent on the nominal ISO sizes and the equations presented in Figure 3 are simply an engineering relationship between two different physical magnitudes. Finally, the aforementioned equations are not universal and should be used exclusively for the tested $\mathrm{Ni}$-Ti endodontic system. For the other $\mathrm{Ni}$-Ti files, linear models with different coefficient values are anticipated.

Although the geometrical configuration and design of the $\mathrm{Ni}-\mathrm{Ti}$ files are important factors in controlling the clinical performance of these micro-instruments, the assessment of their clinical performance with experimental testing remains 
questionable 7,26 . Some studies provide results with great differences, not only among the different brands but also within one brand and type. The main weakness of the currently available experimental protocols is that the clinical performance of the endodontic files has been biased as to the influence of various factors and has been determined separately ${ }^{7,26}$. On the other hand, the results of the current study do not have any immediate clinical implications, as they must be combined with further experimental results of the clinical properties (such as cutting efficiency, loading of dental chips, etc.) in order to determine any possible correlation. For instance, it is logical to assume that a file with a greater surface area has the increased ability of loading dental chips but this requires experimental documentation.

The surface area and volume between the successive files showed significant differences and thus there is no overlap between the successive file numbers, as occurs with the first diameter (d1) below the tip ${ }^{25,30}$. As expected, both the surface area and volume show an increase towards the higher file sizes. The percentage difference between $N^{\circ} 25 / N^{\circ} 30(16.6 \%)$ is almost double the value of No $20 / N^{\circ} 25$ (8.9\%) files sizes, whereas the percentage increase in volume was similar (21.1\% and $17.2 \%$ for No 20/No 25 and No 25/No 30 , respectively) showing a completely different increasing pattern. It should be mentioned that the percentage differences in the surface area show an abrupt increase towards the larger file sizes, while volumetric changes exhibit a declining trend. This implies that the surface area and volume progress towards larger files with a completely different pattern, a finding reflected by the values of the specific surface area (Figure 3 ). The latter is a property that defines the total surface area per unit of bulk volume and has significant importance in cases where the surface area has a significant effect on the system studied. The No 20 files have the highest specific surface area, indicating that more surface area is exposed per volume unit than in $\mathrm{N}^{\circ}$ 25 and No 30 sizes. This value is characteristic of the geometrical design and it is expected to vary significantly among different file designs. Files with a non-standard taper (i.e., ProTaper) are anticipated to demonstrate increased specific surface area due to the significant reduction in file volume.

It is noteworthy that beyond the estimation of the surface area and volume of the scanned files, the 2D and 3D analysis of the scanned files provides tremendous opportunities. Firstly, all the geometrical features (i.e., cutting angle, depth of flutes, crosssectional diameter) measured in longitudinal and cross-section analyses, with optical and electron microscopy can be readily measured with $\mathrm{mXCT}$ methodology. This analysis is advantageous over traditional techniques, as it is non-destructive and thus there is no need for specimen preparation (embedding, cutting, metallographic polishing, conductive coating). Computational analysis of 3D models can be used to quantify any selected geometrical feature for any region of interest. For instance, the real surface area of a file working in a root canal can be easily estimated, selecting the relative region of interest. Secondly, 3D modeling of scanned files can provide more accurate models ${ }^{12}$ for subsequent finite element analyses ${ }^{1}$. On the other hand, the drawbacks of mXCT methodology are the inferior resolution compared to optical and electron microscopy and prolonged scanning times for the model reconstruction in high isotropic resolution. Almost 16 hours of scanning were required in this study for each file. Another limitation is that the image quality might be affected by the beam hardening phenomena. Normally, an $X$-ray beam contains photons with a vast range of energies. In physical metallurgy, the beam hardening phenomenon refers to the absorption of lower energy photons during the passing of a beam through a metal object and once this happens, "beam hardening" occurs as the mean energy of a beam increases. In this study, to minimize the beam hardening effect an Al filter was utilized and a beam hardening correction routine was applied. The former was used to pre-harden the beam by cutting the lower energy photons, while the latter is a correction algorithm which was applied during the reconstruction process. The estimation of the surface area and volume of endodontic files might provide a new approach for the characterization of clinically-related properties such as cutting efficiency, loading of dentin debris etc. This study demonstrates that $\mathrm{mXCT}$ is a powerful tool with interesting applications for endodontic research. Continuing technological advancements allowing development of more powerful mXCT scanners should accelerate the applications of this methodology in the near future.

\section{CONCLUSIONS}

The surface area and volume demonstrated an almost linear increase, while the specific surface area demonstrated an abrupt decrease towards the higher file sizes. For the first time, we have shown that $\mathrm{mXCT}$ is capable of discriminating the quantitative differences in the geometrical properties of the successive sizes of endodontic files. This type of analysis should be used to investigate the unexplored correlations of the various clinical properties of the geometrical characteristics of the endodontic files, opening new avenues for research in the field of endodontics. 


\section{ACKNOWLEDGMENT}

The authors would like to thank the Research Group Program for funding this research project. This study has been funded by a research grant (\# RGP-VPP-206) from the Research Group Program, Deanship of Scientific Research, King Saud University, Riyadh, Saudi Arabia.

\section{REFERENCES}

1- Arbab-Chirani R, Chevalier V, Arbab-Chirani S, Calloch S. Comparative analysis of torsional and bending behavior through finite-element models of $5 \mathrm{Ni}-\mathrm{Ti}$ endodontic instruments. Oral Surg Oral Med Oral Pathol Oral Radiol Endod. 2011;111:115-21. 2- Bergmans L, Van Cleynenbreugel J, Wevers M, Lambrechts P. A methodology for quantitative evaluation of root canal instrumentation using micro-computed tomography. Int Endod J. 2001;34:390-8.

3- Darabara M, Bourithis L, Zinelis S, Papadimitriou GD. Assessment of elemental composition, microstructure, and hardness of stainless steel endodontic files and reamers. J Endod. 2004;30:523-6.

4- De Vos W, Casselman J, Swennen GR. Cone-beam computerized tomography (CBCT) imaging of the oral and maxillofacial region: a systematic review of the literature. Int J Oral Maxillofac Surg. 2009;38:609-25.

5- De Witte Y, Cnudde V, Pieters K, Masschaele B, Dierick M, Vlassenbroeck J, et al. X-ray micro-CT applied to natural building materials and art objects. X-Ray Spectrom. 2008;37:383-7.

6- Dowker SE, Davis GR, Elliott JC. X-ray micro-tomography: nondestructive three-dimensional imaging for in vitro endodontic studies. Oral Surg Oral Med Oral Pathol Oral Radiol Endod. 1997;83:510-6.

7- Himel VT, McSpadden JT, Goodis HE. Instruments, materials and devices. In: Cohen S, Hargreaves KM, editors. Pathways of the pulp. $9^{\text {th }}$ ed. St Louis: Mosby; 2005. p. 521-73.

8- Hübscher W, Barbakow F, Peters OA. Root-canal preparation with FlexMaster: canal shapes analyzed by micro-computed tomography. Int Endod J. 2003;36:740-7.

9- International Organization for Standardization. 3630-1: Dentistry - Root-canal instruments - Part 1: General requirements and test methods. $2^{\text {nd }}$ ed. Geneva: ISO; 2008.

10- Kalender WA. X-ray computed tomography. Phys Med Biol. 2006;51:R29-43.

11- Loizides AL, Kakavetsos VD, Tzanetakis GN, Kontakiotis EG, Eliades G. A comparative study of the effects of two nickel-titanium preparation techniques on root canal geometry assessed by microcomputed tomography. J Endod. 2007;33:1455-9.

12- Magne P. Efficient 3D finite element analysis of dental restorative procedures using micro-CT data. Dent Mater. 2007; 23:539-48.

13- Monteiro PJ, Kirchheim AP, Chae S, Fischer P, MacDowell AA, Schaible $E$, et al. Characterizing the nano and micro structure of concrete to improve its durability. Cement Concrete Comp. 2009;31:577-84.
14- Nielsen RB, Alyassin AM, Peters DD, Carnes DL, Lancaster J. Micro-computed tomography: an advanced system for detailed endodontic research. J Endod. 1995;21:561-8.

15- Paqué F, Peters OA. Micro-computed tomography evaluation of the preparation of long oval root canals in mandibular molars with the self-adjusting file. J Endod. 2011;37:517-21.

16- Parashos $\mathrm{P}$, Messer $\mathrm{HH}$. Rotary NiTi instrument fracture and its consequences. J Endod. 2006;32:1031-43.

17- Peters OA, Laib A, Göhring TN, Barbakow F. Changes in root canal geometry after preparation assessed by high-resolution computed tomography. J Endod. 2001;27:1-6.

18- Peters OA, Laib A, Rüegsegger P, Barbakow F. Threedimensional analysis of root canal geometry by high-resolution computed tomography. J Dent Res. 2000;79:1405-9.

19- Peters OA, Paqué F. Root canal preparation of maxillary molars with the self-adjusting file: a micro-computed tomography study. J Endod. 2011;37:53-7.

20- Rahimi M, Parashos P. A novel technique for the removal of fractured instruments in the apical third of curved root canals. Int Endod J. 2009;42:264-70.

21- Rhodes JS, Ford TR, Lynch JA, Liepins PJ, Curtis RV. Microcomputed tomography: a new tool for experimental endodontology. Int Endod J. 1999;32:165-70.

22- Schäfer E, Florek H. Efficiency of rotary nickel-titanium K3 instruments compared with stainless steel hand K-Flexofile. Part 1. Shaping ability in simulated curved canals. Int Endod J. 2003;36:199-207.

23- Spanaki-Voreadi AP, Kerezoudis NP, Zinelis S. Failure mechanism of ProTaper $\mathrm{Ni}-\mathrm{Ti}$ rotary instruments during clinical use: fractographic analysis. Int Endod J. 2006;39:171-8.

24- Spili $P$, Parashos $P$, Messer $\mathrm{HH}$. The impact of instrument fracture on the outcome of endodontic treatment. J Endod. 2005;31:845-50.

25- Stenman E, Spångberg LS. Root canal instruments are poorly standardized. J Endod. 1993;19:327-34

26- Viana AC, Chaves Craveiro de Melo M, Guiomar de Azevedo Bahia M, Lopes Buono VT. Relationship between flexibility and physical, chemical, and geometric characteristics of rotary nickeltitanium instruments. Oral Surg Oral Med Oral Pathol Oral Radiol Endod. 2010;110:527-33.

27- Wildey WL, Senia ES, Montgomery S. Another look at root canal instrumentation. Oral Surg Oral Med Oral Pathol. 1992;74:499507.

28- Xu X, Eng M, Zheng Y, Eng D. Comparative study of torsional and bending properties for six models of nickel-titanium root canal instruments with different cross-sections. J Endod. 2006;32:3725.

29- Zinelis S, Eliades T, Eliades G. A metallurgical characterization of ten endodontic $\mathrm{Ni}-\mathrm{Ti}$ instruments: assessing the clinical relevance of shape memory and super-elastic properties of $\mathrm{Ni}-\mathrm{Ti}$ endodontic instruments. Int Endod J. 2010;43:125-34.

30- Zinelis S, Magnissalis EA, Margelos J, Lambrianidis T. Clinical relevance of standardization of endodontic files dimensions according to the ISO 3630-1 specification. J Endod. 2002;28:36770 . 